$62^{\text {ème }}$ Congrès de la SFCO, 02010 (2014)

DOI: $10.1051 /$ sfco/20146202010

(C) Owned by the authors, published by EDP Sciences, 2014

\title{
Traitement du lichen plan buccal érosif cortico-résistant par mycrophénolate mofétil : 3 cas.
}

\author{
Fénelon $\mathbf{M}^{1}$, Boralevi $\mathrm{F}^{2}$, Catros $\mathbf{S}^{1}$, Fricain JC \\ 1 Service d'odontologie CHU Pellegrin, place Amélie-Raba-Léon, 33076, Bordeaux, FRANCE \\ 2 Service de dermatologie CHU St André, 1, rue Jean Burguet, 33075, Bordeaux, FRANCE
}

Le lichen plan est une dermatose inflammatoire chronique, d'étiologie inconnue, évoluant par poussées. II se caractérise sur le plan histologique par un infiltrat lympho-plasmocytaire sous épithélial provoquant la formation de corps apoptotiques (Garcia-Garcia 2012). Le lichen plan buccal érosif se manifeste par des lésions érythémateuses, érosives et ulcérées souvent invalidantes pour le patient et résistantes au traitement. En première intention, le traitement repose sur l'application topique de corticostéroïdes (clobétasol) ou plus récemment d'inhibiteurs de la calcineurine (resende 2013). En deuxième intention, si la réponse est insuffisante, un traitement par corticostéroïde (prednisolone) per os peut être instauré, associé ou non à un immunossupresseur (azathioprine, ciclosporine) (Manousaridis 2013). Ces traitements peuvent se révéler décevants ou difficiles à poursuivre sur le long terme en raison de leurs effets secondaires. Le mycophénolate mofétil est un immunosuppresseur dont l'intérêt dans le traitement des lichens plans oraux a été rapporté chez seulement 11 patients (Frieling 2003, Dalmau 2007, Wee 2010). Nous rapportons 3 nouveaux cas cliniques montrant l'intérêt du mycophénolate mofétil dans la prise en charge du lichen plan oral cortico résistant.

Les trois patients présentaient un lichen plan buccal érosif, sévère, invalidant et réfractaire aux thérapeutiques conventionnelles. La première patiente âgée de 60 ans présentait un lichen plan pluri focal avec atteintes vulvaire, buccale et oesophagienne. Une corticothérapie de $1 / 2 \mathrm{mg} / \mathrm{kg}$ avait été instaurée pendant 6 moismais l'atteinte buccale persistait. La deuxième patiente âgée de 88 ans était atteinte d'un lichen plan buccal érosif de l'ensemble des muqueuses buccales.
Elle avait eu des traitements locaux par clobétasol et tacrolimus inefficaces. Deux corticothérapies par voie générale $(1 \mathrm{mg} / \mathrm{kg}$ pendant 3 mois puis diminution progressive de la posologie sur un an) ont été prescrites avec un phénomène de rebond à l'arrêt. Le troisième patient âgé de 58 ans présentait un lichen plan érosif jugal interne bilatéral, douloureux et toujours invalidant malgré l'application locale de clobétasol puis de sirolimus. Un traitement par mycophenolate mofétil a été introduit à raison de $2 \mathrm{~g} / \mathrm{j}$ chez ces trois patients. Une bonne tolérance au traitement a été observée chez les trois patients.Seul le troisième patient a présenté des épisodes infectieux $0 R L$ itératifs éventuellement imputables au mycophenolate mofétil. La diminution de la posologie à $1,5 \mathrm{~g} / \mathrm{j}$ a permis la poursuite du traitement.

Une rémission totale a été notée dans le premier cas après 2 ans de traitement et un recul de trois ans. Une rémission partielle importante a été observée dans les 2 autres cas. Ces résultats corroborent les résultats publiés (Frieling \& coll 2003,Dalmau \& coll 2007, Wee \& coll 2010).

Le mycophénolate mofétil semble être un traitement efficace des lichens plan disséminés et érosifs réfractaires aux thérapeutiques conventionnelles. La bonne tolérance pourrait permettre d'envisager son utilisation en dehors des situations de dernier recours.

FENELON Mathilde mathildefenelon@live.fr

This is an Open Access article distributed under the terms of the Creative Commons Attribution License 4.0, which permits unrestricted use, distribution, and reproduction in any medium, provided the original work is properly cited. 